\title{
MINLP cost optimization of industrial steel building
}

\author{
S. Kravanja \& T. Žula \\ University of Maribor, Faculty of Civil Engineering, \\ Maribor, Slovenia
}

\begin{abstract}
This paper presents the cost optimization of a single-storey industrial steel building structure. The optimization is performed by the Mixed-Integer Non-linear programming approach, MINLP. The structure consists of the main portal frames, which are mutually connected with the purlins. All structural elements are proposed to be built up of standard hot rolled I sections. The MINLP performs the simultaneous cost, topology and discrete sizes optimization of the building structure. Since the discrete/continuous optimization problem is non-convex and highly non-linear, the Modified Outer-Approximation/EqualityRelaxation (OA/ER) algorithm has been used for the optimization. Alongside the optimal structure's costs, the optimal number of main portal frames and purlins as well as all standard cross-section sizes have been obtained.

Keywords: structural optimization, cost optimization, topology optimization, discrete sizes optimization, mixed-integer non-linear programming, MINLP, industrial building.
\end{abstract}

\section{Introduction}

This paper deals with the simultaneous cost, topology and standard discrete sizes optimization of single-storey industrial steel building structure. The optimization is performed by the Mixed-Integer Non-linear Programming, MINLP. The MINLP is a combined discrete and continuous optimization technique. The MINLP handles with continuous and discrete binary 0-1 variables simultaneously. While continuous variables are defined for the continuous optimization of parameters (dimensions, stresses, strains, weights, costs, etc.), discrete variables are used to express discrete decisions, i.e. usually the existence 
or non-existence of structural elements inside the defined structure. Different materials and standard dimensions may also be defined as discrete alternatives. Since continuous and discrete optimizations are carried out simultaneously, the MINLP approach also finds optimal continuous parameters (e.g. structural costs), structural topology and discrete standard cross-sizes simultaneously.

The considered steel building structures are consisted from the main portal frames, which are mutually connected with the purlins. All structural elements are proposed to be built up of standard hot rolled I sections. The MINLP discrete/continuous optimization problems of such sceletal structures are in most cases comprehensive, non-convex and highly non-linear. It is proposed that the optimization will be performed through three steps. The first one includes the generation of a mechanical superstructure of different topology and standard sizes alternatives, the second one involves the development of an MINLP model formulation and the last one consists of a solution for the defined MINLP optimization problem. The Modified Outer-Approximation/Equality-Relaxation algorithm is used to perform the optimization, see Kravanja and Grossmann [1], Kravanja et al. [2-4].

The objective of the optimization is to minimize the self-manufacturing costs of the single-storey industrial building. The defined cost objective function comprises material costs, anti-corrosion protection painting costs, steel cutting costs as well as assembling and erection costs of the structure. The cost objective function is subjected to the set of the equality and inequality constraints known from the structural analysis. The dimensioning of steel members is performed in accordance with Eurocode 3 [5].

A single-phase MINLP costs optimization is proposed. It starts with the continuous NLP optimization of a relative high defined structural topology, while standard sizes are relaxed temporary into continuous parameters. When the optimal continuous topology and other parameters are found, standard sizes of cross-sections are re-established and the simultaneous cost, topology and standard sizes optimization of beams, columns and purlins is then continued until the optimal solution is found.

\section{Single-storey industrial steel building}

The paper discusses a single-storey industrial steel building structure, see fig. 1 . The structures are consisted from the main portal frames, mutually connected with the purlins. Columns, beams and purlins are proposed to be built up of steel standard hot rolled I sections.

In the field of the optimization of steel sceletal structures many different optimization methods have been proposed. O'Brien and Dixon [6] have proposed a linear programming approach for the optimal design of pitched roof frames. Guerlement et al. [7] have introduced a practical method for single-storey steel structures, based on a discrete minimum weight design and Eurocode 3 design constraints. Recently, Saka [8] has considered an optimum design of pitched roof steel frames with haunched rafters by using a genetic algorithm. One of the latest 
researches reported in this field is the work of Hernández et al. [9], where authors have considered minimum weight design of steel portal frames with software developed for structural optimization.

This paper introduces the simultaneous cost, topology and standard discrete sizes optimization of a single-storey industrial steel building structure. The optimization is performed by the Mixed-Integer Non-linear Programming, MINLP. The objective of the optimization is to minimize the structure's selfmanufacturing costs.

The considered sceletal structure is analysed under the combined effects of the self-weight of frame members, uniformly distributed surface variable load (snow and wind), concentrated horizontal variable load (wind) and an initial frame imperfection. The purlins are designed to transfer permanent load (selfweight of purlins and weight of roof) and variable load (snow and wind) to frame structures.

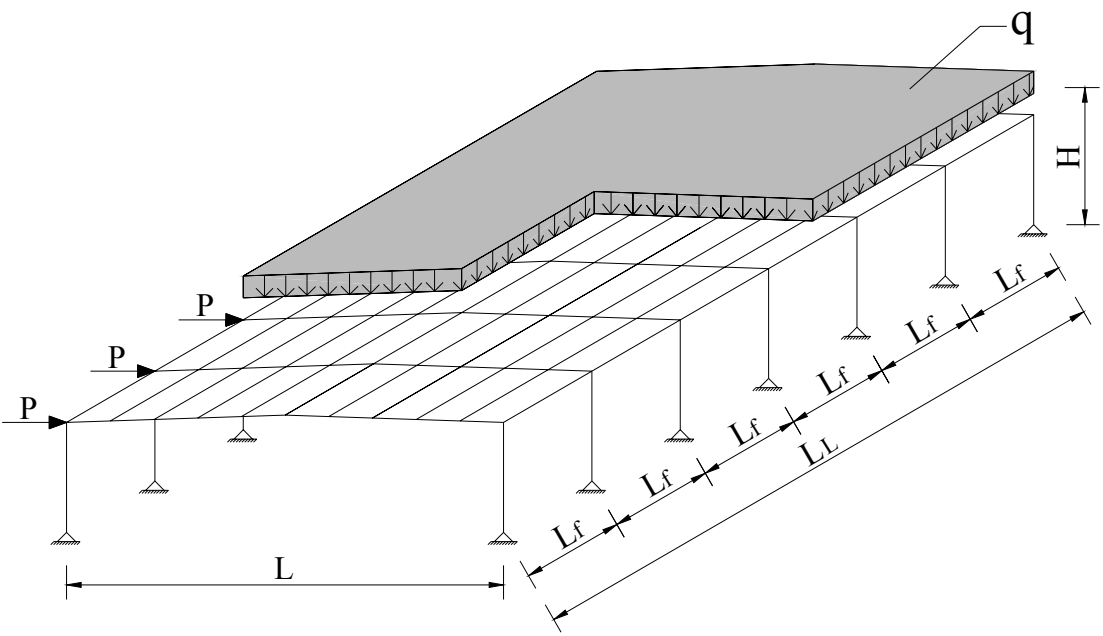

Figure 1: Single-storey industrial building.

Internal forces are calculated by the elastic first-order analysis. The dimensioning of steel members is performed in accordance with Eurocode 3 for the conditions of both ultimate limit and serviceability limit states.

When the ultimate limit state of structural members is considered, the elements are checked for axial resistance, shear resistance, bending moment resistance, interaction between bending moment and axial force, axial compression/buckling resistance, lateral buckling resistance as well as for the interaction between axial compression/buckling and lateral buckling resistance moment.

Considering the serviceability limit state, the vertical deflections of beams and purlins are calculated and checked. The total deflection $\delta_{\max }$ subjected to the overall load and the deflections $\delta_{2}$ subjected to the variable imposed load are 
calculated to be smaller than limited maximum values: span/200 and span/250, respectively. The horizontal deflections $\Delta$ are also checked for the recommended limits: the relative horizontal deflections should be smaller then the height/150 of each portal frame.

\section{MINLP model formulation for mechanical superstructures}

It is assumed that a general nonconvex and nonlinear discrete/continuous optimization problem can be formulated as an MINLP problem (MINLP-G) in the form:

$$
\begin{gathered}
\min \quad z=\boldsymbol{c}^{T} \boldsymbol{y}+f(\boldsymbol{x}) \\
\text { s.t. } \quad \boldsymbol{h}(\boldsymbol{x})=\boldsymbol{0} \\
\boldsymbol{g}(\boldsymbol{x}) \leq \boldsymbol{0} \\
\boldsymbol{B} \boldsymbol{y}+\boldsymbol{C} \boldsymbol{x} \leq \boldsymbol{b} \\
\boldsymbol{x} \in X=\left\{\boldsymbol{x} \in R^{n}: \boldsymbol{x}^{\mathrm{LO}} \leq \boldsymbol{x} \leq \boldsymbol{x}^{\mathrm{UP}}\right\} \\
\boldsymbol{y} \in Y=\{0,1\}^{m}
\end{gathered}
$$

where $\boldsymbol{x}$ is a vector of continuous variables specified in the compact set $X$ and $\boldsymbol{y}$ is a vector of discrete, mostly binary $0-1$ variables. Functions $f(\boldsymbol{x}), \boldsymbol{h}(\boldsymbol{x})$ and $\boldsymbol{g}(\boldsymbol{x})$ are nonlinear functions involved in the objective function $z$, equality and inequality constraints, respectively. Finally, $\boldsymbol{B} \boldsymbol{y}+\boldsymbol{C} \boldsymbol{x} \leq \boldsymbol{b}$ represents a subset of mixed linear equality/inequality constraints.

The above general MINLP model formulation has been adapted for the optimization of mechanical superstructures (MINLP-SMS). The resulted formulation is more specific, particularly in variables and constraints. It can be used also for the modelling the steel industrial building. It is given in the following form:

$$
\begin{gathered}
\min \quad z=\boldsymbol{c}^{T} \boldsymbol{y}+f(\boldsymbol{x}) \\
\text { s.t. } \quad \boldsymbol{h}(\boldsymbol{x})=\boldsymbol{0} \\
\boldsymbol{g}(\boldsymbol{x}) \leq \boldsymbol{0} \\
\boldsymbol{A}(\boldsymbol{x}) \leq \boldsymbol{a} \\
\boldsymbol{E} \boldsymbol{y} \leq \boldsymbol{e} \\
\boldsymbol{D y}^{e}+\boldsymbol{R}(\boldsymbol{x}) \leq \boldsymbol{r} \\
\boldsymbol{K} \boldsymbol{y}^{e}+\boldsymbol{L}\left(\boldsymbol{d}^{c n}\right) \leq \boldsymbol{k} \\
\boldsymbol{P} \boldsymbol{y}+\boldsymbol{S}\left(\boldsymbol{d}^{s t}\right) \leq \boldsymbol{s} \\
\boldsymbol{x} \in X=\left\{\boldsymbol{x} \in R{ }^{n}: \boldsymbol{x}^{\mathrm{LO}} \leq \boldsymbol{x} \leq \boldsymbol{x}^{\mathrm{UP}}\right\} \\
\boldsymbol{y} \in Y=\{0,1\}^{m}
\end{gathered}
$$


The MINLP model formulation for mechanical superstructures is proposed to be described as follows:

- Included are continuous variables $\boldsymbol{x}=\{\boldsymbol{d}, \boldsymbol{p}\}$ and discrete binary variables $\boldsymbol{y}=\left\{\boldsymbol{y}^{e}, \boldsymbol{y}^{s t}\right\}$. Continuous variables are partitioned into design variables $\boldsymbol{d}=\left\{\boldsymbol{d}^{c n}\right.$, $\left.\boldsymbol{d}^{\text {st }}\right\}$ and into performance (nondesign) variables $\boldsymbol{p}$, where subvectors $\boldsymbol{d}^{c n}$ and $\boldsymbol{d}^{s t}$ stand for continuous and standard dimensions, respectively. Subvectors of binary variables $\boldsymbol{y}^{e}$ and $\boldsymbol{y}^{s t}$ denote the potential existence of structural elements inside the superstructure (the topology determination) and the potential selection of standard dimension alternatives, respectively.

- The economical objective function $z$ involves fixed cost charges in the linear term $\boldsymbol{c}^{\mathrm{T}} \boldsymbol{y}$ and dimension dependant costs in the term $f(\boldsymbol{x})$.

- Parameter nonlinear and linear constraints $\boldsymbol{h}(\boldsymbol{x})=\mathbf{0}, \boldsymbol{g}(\boldsymbol{x}) \leq \mathbf{0}$ and $\boldsymbol{A}(\boldsymbol{x}) \leq \boldsymbol{a}$ represent the rigorous system of the design, loading, stress, deflection, stability, etc. constraints known from the structural analysis.

- Integer linear constraints $\boldsymbol{E} \boldsymbol{y} \leq \boldsymbol{e}$ are proposed to describe relations between binary variables.

- Mixed linear constraints $\boldsymbol{D y}^{e}+\boldsymbol{R}(\boldsymbol{x}) \leq \boldsymbol{r}$ restore interconnection relations between currently selected or existing structural elements (corresponding $\left.y^{e}=1\right)$ and cancel relations for currently disappearing or nonexisting elements (corresponding $y^{e}=0$ ).

- Mixed linear constraints $\boldsymbol{K y}^{e}+\boldsymbol{L}\left(\boldsymbol{d}^{c n}\right) \leq \boldsymbol{k}$ are proposed to define the continuous design variables for each existing structural element. The space is defined only when the corresponding structure element exists $\left(y^{e}=1\right)$, otherwise it is empty.

- Mixed linear constraints $\boldsymbol{P} \boldsymbol{y}+\boldsymbol{S}\left(\boldsymbol{d}^{s t}\right) \leq \boldsymbol{s}$ define standard discrete design variables $\boldsymbol{d}^{s t}$. Each standard discrete dimension $d^{s t}$ is determined as a scalar product between its vector of standard discrete dimension constants $\boldsymbol{q}$ and its vector of binary variables $\boldsymbol{y}^{s t}$, eqn. (1). Only one discrete value can be selected for each standard dimension since the sum of binary variables must be equal one, eqn. (2):

$$
\begin{gathered}
d^{\mathrm{st}}=\sum_{i \in I} q_{i} y_{i}^{\mathrm{st}} \\
\sum_{i \in I} y_{i}^{\mathrm{st}}=1
\end{gathered}
$$

\section{The optimization model}

A special MINLP optimization model for a single-storey industrial steel building structure has been developed. The model is constructed on the basis of the mentioned MINLP-SMS special model formulation for mechanical superstructures. As an interface for mathematical modelling and data inputs/outputs GAMS (General Algebraic Modelling System), a high level language, is used [10]. 
The optimization model for the steel structure contains the cost objective function, which is subjected to the (in)equality constraints. The variables include continuous variables for dimensions, internal forces, deflections, costs, etc. as well as discrete binary $0-1$ variables for the topology and standard sizes determination. Equality and inequality constraints represent a rigorous system of the design, loading, resistance, stress, deflections and stability functions. The dimensioning constraints are determined according to Eurocode 3 (ultimate and serviceability limit states).

The defined cost objective function, see eqn. (3), comprises material costs, anti-corrosion protection painting costs, steel cutting costs as well as assembling and erection costs of the structure:

$\min :$

$\operatorname{COST}=\sum_{i} \operatorname{Vol}_{i} \times \rho \times C_{\text {mat }}+\sum_{i} A_{i} \times C_{\text {paint }}+\sum_{i} L c u t_{i} \times C_{\text {cut }}+\sum_{i}\left(y_{i} \mid y_{i}=1\right) \times C_{\text {erect }}$

where COST [EUR] represents the self-manufacturing costs of the building structure, $\Sigma_{i} \mathrm{Vol}_{i}\left[\mathrm{~m}^{3}\right]$ stands for the volumes of $i, i \in I$, structural members of the building, $\rho$ denotes steel density $7850 \mathrm{~kg} / \mathrm{m}^{3}, C_{m a t}$ is the price of the structural steel [EUR $/ \mathrm{kg}] ; \Sigma_{i} A_{i}\left[\mathrm{~m}^{2}\right]$ stands for the surface areas of $i$ steel members, $C_{\text {paint }}$ $\left[\mathrm{EUR} / \mathrm{m}^{2}\right]$ is the price of the anti-corrosion and fire (R 30) protection painting; $\Sigma_{i} L_{c u t}[\mathrm{~m}]$ represents steel cutting lengths of $i$ members, $C_{c u t}[\mathrm{EUR} / \mathrm{m}]$ is the price of the steel cutting; $\Sigma_{i}\left(y_{i} \mid y_{i}=1\right)$ denotes a sum of non-zero binary variables which represents the number of the existing structural elements $=$ the building topology, and $C_{\text {erect }}$ [EUR] is the price of assembling end erection of each $i$-th building element, i.e. each portal frame and purlin.

\section{MINLP optimization}

The Modified Outer-Approximation/Equality-Relaxation (OA/ER) algorithm by Kravanja and Grossmann [1] was used to perform the MINLP optimization. The OA/ER algorithm consists of solving an alternative sequence of Non-linear Programming optimization subproblems (NLP) and Mixed-Integer Linear Programming master problems (MILP). The former corresponds to the optimization of parameters for a sceletal structure with fixed topology and standard sizes and yields an upper bound to the objective to be minimized. The latter involves a global approximation to the superstructure of alternatives in which a new topology and standard sizes are identified so that its lower bound does not exceed the current best upper bound. The search of a convex problem is terminated when the predicted lower bound exceeds the upper bound, otherwise it is terminated when the NLP solution can be improved no more. The OA/ER algorithm guarantees the global optimality of solutions for convex and quasiconvex optimization problems.

The OA/ER algorithm as well as all other mentioned MINLP algorithms do not generally guarantee that the solution found is the global optimum. This is due to the presence of nonconvex functions in the models that may cut off the global optimum. In order to reduce undesirable effects of nonconvexities the following 
modifications are applied for the master problem of the OA/ER algorithm: the deactivation of linearizations, the decomposition and the deactivation of the objective function linearization, the use of the penalty function, the use of the upper bound on the objective function to be minimized as well as the global convexity test and the validation of the outer approximations.

A single-phase MINLP is proposed for the optimization. It starts with the continuous NLP optimization of a relative high defined structural topology (with a high number of portal frames and purlins), while standard sizes are relaxed temporary into continuous parameters. When the optimal continuous topology and other parameters are found, standard sizes of cross-sections are reestablished and the simultaneous discrete/continuous cost, topology and standard sizes optimization of beams, columns and purlins is then continued until the optimal solution is found.

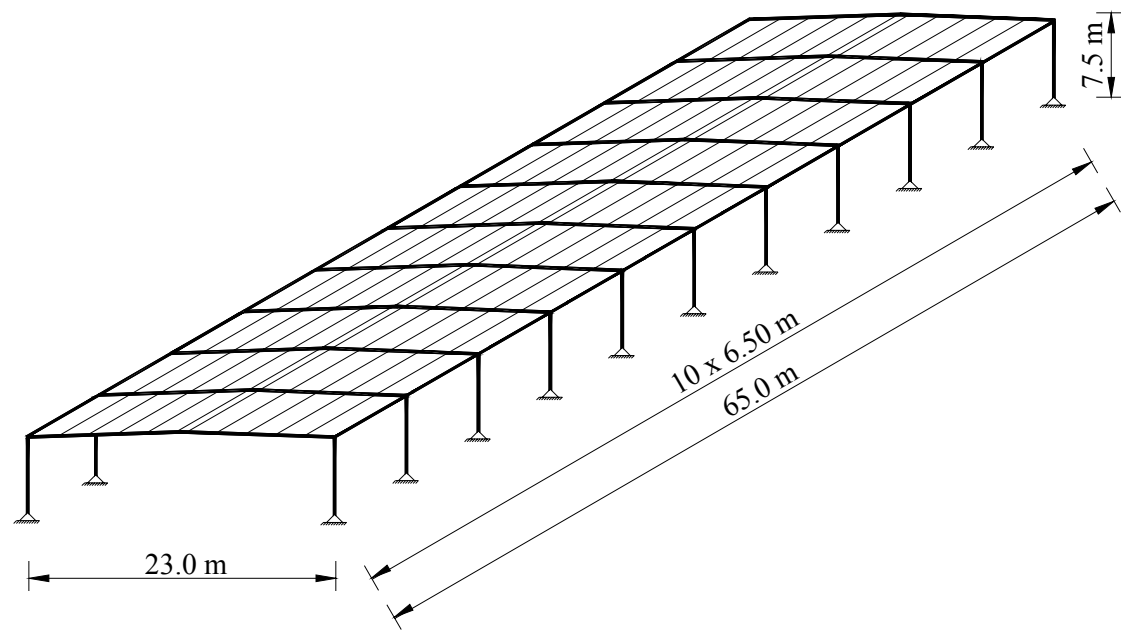

Figure 2: Optimal design of the single-storey industrial building.

\section{The example}

The paper presents an example of the cost, topology and standard sizes optimization of a single-storey industrial building. The building is $23 \mathrm{~m}$ wide, $65 \mathrm{~m}$ long and $7 \mathrm{~m}$ high, see fig. 2. The structure is consisted from equal non-sway steel portal frames which are mutually connected with the purlins.

The portal frame is subjected to self-weight $g$ (structure plus roof), uniformly distributed surface variable load $q$ (snow $s$ and vertical wind $w_{\text {vert }}$ ), concentrated variable load $P$ at the top of columns (caused by horizontal wind $w_{\text {horiz }}$ and initial frame imperfection $F \varphi$ ). Variable imposed load $\left(s=1.60 \mathrm{kN} / \mathrm{m}^{2}, w_{\text {vert }}=0.14\right.$ $\mathrm{kN} / \mathrm{m}^{2}$ and $w_{\text {horiz }}=0.60 \mathrm{kN} / \mathrm{m}^{2}$ ) is defined as the uniformly distributed surface load in the model input data. Both, the horizontal concentrated load at the top of the columns and the vertical uniformly distributed line load on the beams are 
calculated through the optimization automatically considering the intermediate distance between the portal frames.

Design/dimensioning was performed in accordance with Eurocode 3. The design loads were calculated for the conditions of both ultimate limit states and serviceability limit states: (a) for ultimate limit states: $1.35 \cdot\left(g+s+w_{\text {vert }}+P\right)$, (b) for serviceability limit states: $1.00 \cdot g+0.90 \cdot\left(s+w_{\text {vert }}+P\right)$. Internal forces and deflections were calculated by the elastic first-order analysis.

The portal frame superstructure was generated in which all possible structures were embedded by 30 portal frame alternatives, 20 purlin alternatives and 24 different alternatives of standard hot rolled European wide flange HEA sections (from HEA 100 to HEA 1000) for each column, beam and purlin separately. The material used was steel S 355.

The optimization was performed by the MINLP optimization approach. The task of the optimization was to find the minimal structure self-manufacturing costs, the optimal topology (the optimal number of portal frames and purlins) and optimal standard sizes. The economical objective function included the material, anti-corrosion and fire ( $\mathrm{R}$ 30) protection painting, steel cutting as well as assembling and erection costs of the structure. The economic data for the optimization are presented in table 1 .

Table 1: Economic data for optimization.

\begin{tabular}{|l|l|c|}
\hline$C_{\text {mat }}$ & price of structural steel S 355 & $1.1 \mathrm{EUR} / \mathrm{kg}$ \\
\hline$C_{\text {paint }}$ & anti-corrosion protecting costs & $22.0 \mathrm{EUR} / \mathrm{m}^{2}$ \\
\hline$C_{\text {cut }}$ & steel cutting costs & $4.0 \mathrm{EUR} / \mathrm{m}$ \\
\hline$C_{\text {erect }}$ & assembling and erection costs & $500.0 \mathrm{EUR} /$ portal frame \\
& & $200.0 \mathrm{EUR} /$ purlin \\
\hline
\end{tabular}

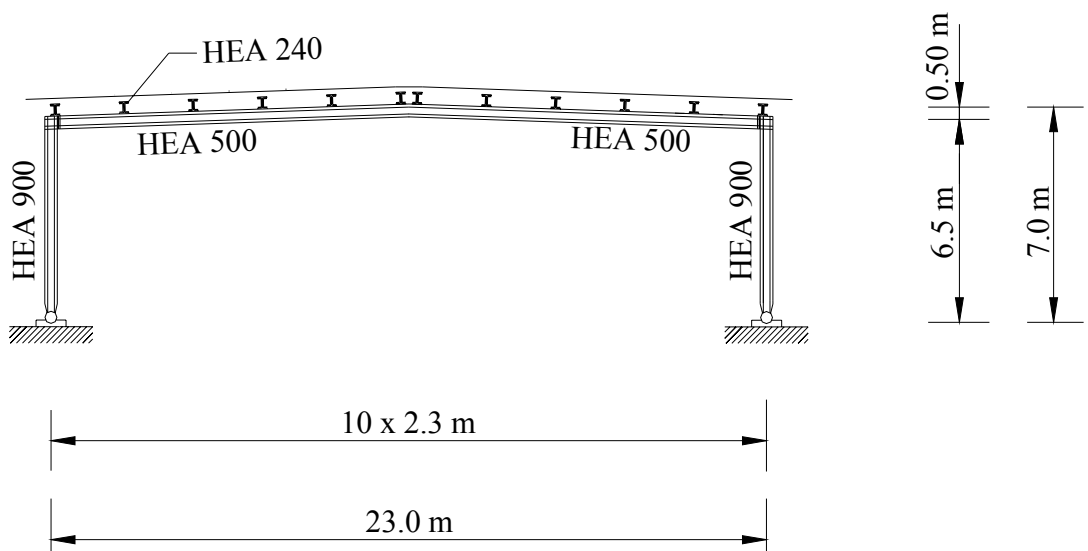

Figure 3: Optimal design of the portal frame. 
The optimization was carried out by a user-friendly version of the MINLP computer package MIPSYN, the successor of PROSYN [1] and TOP [2-4]. As an interface for mathematical modelling GAMS (General Algebraic Modelling System was used [10]. The Modified OA/ER algorithm and the single-phase optimization were applied, where GAMS/CONOPT3 (Generalized reducedgradient method) [11] was used to solve NLP subproblems and GAMS/Cplex 7.0 (Branch and Bound) [12] was used to solve MILP master problems.

The final optimal solution of 189039 EUR was obtained in the $3^{\text {rd }}$ main MINLP iteration. The optimal solution represents the obtained »minimal« self material and labour costs of the considered steel industrial building structure. The selling price may be at least twice higher. The solution also comprises the calculated structure mass of 122.42 tons, the building topology of 11 portal frames and 12 purlins, fig. 2, and all standard sizes of columns, beams and purlins, fig. 3.

\section{Conclusions}

The paper presents the simultaneous cost, topology and standard sizes optimization of the single-storey industrial steel building structure. It is proposed that the optimization will be performed by the Mixed-Integer Non-linear Programming (MINLP) approach. The MINLP was found to be a successful optimization technique for solving this type of structures.

\section{References}

[1] Kravanja, Z. and Grossmann, I.E., New Developments and Capabilities in PROSYN - An Automated Topology and Parameter Process Synthesizer, Computers chem. Eng., 18, pp. 1097-1114, 1994.

[2] Kravanja, S., Kravanja, Z. and Bedenik, B.S., The MINLP optimization approach to structural synthesis. Part I: A general view on simultaneous topology and parameter optimization, Int. J. Numer. Methods Eng. 43, pp. 263-292, 1998.

[3] Kravanja, S., Kravanja, Z. and Bedenik, B.S., The MINLP optimization approach to structural synthesis. Part II: Simultaneous topology, parameter and standard dimension optimization by the use of the Linked two-phase MINLP strategy, Int. J. Numer. Methods Eng. 43, pp. 293-328, 1998.

[4] Kravanja, S., Kravanja, Z. and Bedenik, B.S., The MINLP optimization approach to structural synthesis. Part III: Synthesis of roller and sliding hydraulic steel gate structures, Int. J. Numer. Methods Eng. 43, pp. 329364, 1998.

[5] Eurocode 3, Design of steel structures, European Committee for Standardization, 1992.

[6] O'Brien, E.J. and Dixon, A.S., Optimal plastic design of pitched roof frames for multiple loading, Comput. Struct. 64, pp. 737-740, 1997. 
118 Computer Aided Optimum Design in Engineering X

[7] Guerlement, G., Targowski, R., Gutkowski, W., Zawidzka J. and Zawidzki, J., Discrete minimum weight design of steel structures using EC3 code, Struct. Multidisc. Optim. 22, pp. 322-327, 2001.

[8] Saka, M.P., Optimum design of pitched roof steel frames with haunched rafters by genetic algorithm, Comput. Struct. 81, pp. 1967-1978, 2003.

[9] Hernández, S., Fontán, A.N., Perezzán, J.C. and Loscos, P., Design optimization of steel portal frames, Adv. Eng. Software. 36, pp. 626-633, 2005.

[10] Brooke, A., Kendrick, D. and Meeraus, A., GAMS - A User's Guide, Scientific Press, Redwood City, CA, 1988.

[11] Drudd, A.S., CONOPT - A Large-Scale GRG Code, ORSA J. Comput. 6, pp. 207-216, 1994.

[12] CPLEX User Notes, ILOG inc. 